\title{
Data analysis in qualitative research
}

Unquestionably, data analysis is the most complex and mysterious of all of the phases of a qualitative project, and the one that receives the least thoughtful discussion in the literature. For neophyte nurse researchers, many of the data collection strategies involved in a qualitative project may feel familiar and comfortable. After all, nurses have always based their clinical practice on learning as much as possible about the people they work with, and detecting commonalities and variations among and between them in order to provide individualised care. However, creating a database is not sufficient to conduct a qualitative study. In order to generate findings that transform raw data into new knowledge, a qualitative researcher must engage in active and demanding analytic processes throughout all phases of the research. Understanding these processes is therefore an important aspect not only of doing qualitative research, but also of reading, understanding, and interpreting it.

For readers of qualitative studies, the language of analysis can be confusing. It is sometimes difficult to know what the researchers actually did during this phase and to understand how their findings evolved out of the data that were collected or constructed. Furthermore, in describing their processes, some authors use language that accentuates this sense of mystery and magic. For example, they may claim that their conceptual categories "emerged" from the data ${ }^{1}$-almost as if they left the raw data out overnight and awoke to find that the data analysis fairies had organised the data into a coherent new structure that explained everything! In this EBN notebook, I will try to help readers make sense of some of the assertions that are made about qualitative data analysis so that they can develop a critical eye for when an analytical claim is convincing and when it is not.

\section{Qualitative data}

Qualitative data come in various forms. In many qualitative nursing studies, the database consists of interview transcripts from open ended, focused, but exploratory interviews. However, there is no limit to what might possibly constitute a qualitative database, and increasingly we are seeing more and more creative use of such sources as recorded observations (both video and participatory), focus groups, texts and documents, multi-media or public domain sources, policy manuals, photographs, and lay autobiographical accounts.

Qualitative data are not the exclusive domain of qualitative research. Rather, the term can refer to anything that is not quantitative, or rendered into numerical form. Many quantitative studies include open ended survey questions, semistructured interviews, or other forms of qualitative data. What distinguishes the data in a quantitative study from those generated in a qualitatively designed study is a set of assumptions, principles, and even values about truth and reality. Quantitative researchers accept that the goal of science is to discover the truths that exist in the world and to use the scientific method as a way to build a more complete understanding of reality. Although some qualitative researchers operate from a similar philosophical position, most recognise that the relevant reality as far as human experience is concerned is that which takes place in subjective experience, in social context, and in historical time. Thus, qualitative researchers are often more concerned about uncovering knowledge about how people think and feel about the circumstances in which they find themselves than they are in making judgements about whether those thoughts and feelings are valid.

\section{Qualitative analytic reasoning processes}

What makes a study qualitative is that it usually relies on inductive reasoning processes to interpret and structure the meanings that can be derived from data. Distinguishing inductive from deductive inquiry processes is an important step in identifying what counts as qualitative research. Generally, inductive reasoning uses the data to generate ideas (hypothesis generating), whereas deductive reasoning begins with the idea and uses the data to confirm or negate the idea (hypothesis testing). ${ }^{2}$ In actual practice, however, many quantitative studies involve much inductive reasoning, whereas good qualitative analysis often requires access to a full range of strategies. ${ }^{3}$ A traditional quantitative study in the health sciences typically begins with a theoretical grounding, takes direction from hypotheses or explicit study questions, and uses a predetermined (and auditable) set of steps to confirm or refute the hypothesis. It does this to add evidence to the development of specific, causal, and theoretical explanations of phenomena. ${ }^{3}$ In contrast, qualitative research often takes the position that an interpretive understanding is only possible by way of uncovering or deconstructing the meanings of a phenomenon. Thus, a distinction between explaining how something operates (explanation) and why it operates in the manner that it does (interpretation) may be a more effective way to distinguish quantitative from qualitative analytic processes involved in any particular study.

Because data collection and analysis processes tend to be concurrent, with new analytic steps informing the process of additional data collection and new data informing the analytic processes, it is important to recognise that qualitative data analysis processes are not entirely distinguishable from the actual data. The theoretical lens from which the researcher approaches the phenomenon, the strategies that the researcher uses to collect or construct data, and the understandings that the researcher has about what might count as relevant or important data in answering the research question are all analytic processes that influence the data. Analysis also occurs as an explicit step in conceptually interpreting the data set as a whole, using specific analytic strategies to transform the raw data into a new and coherent depiction of the thing being studied. Although there are many qualitative data analysis computer programs available on the market today, these are essentially aids to sorting and organising sets of qualitative data, and none are capable of the intellectual and conceptualising processes required to transform data into meaningful findings.

\section{Specific analytic strategies}

Although a description of the actual procedural details and nuances of every qualitative data analysis strategy is well beyond the scope of a short paper, a general appreciation of the theoretical assumptions underlying some of the more common approaches can be helpful in understanding what a researcher 
is trying to say about how data were sorted, organised, conceptualised, refined, and interpreted.

\section{CONSTANT COMPARATIVE ANALYSIS}

Many qualitative analytic strategies rely on a general approach called "constant comparative analysis". Originally developed for use in the grounded theory methodology of Glaser and Strauss, ${ }^{4}$ which itself evolved out of the sociological theory of symbolic interactionism, this strategy involves taking one piece of data (one interview, one statement, one theme) and comparing it with all others that may be similar or different in order to develop conceptualisations of the possible relations between various pieces of data. For example, by comparing the accounts of 2 different people who had a similar experience, a researcher might pose analytical questions like: why is this different from that? and how are these 2 related? In many qualitative studies whose purpose it is to generate knowledge about common patterns and themes within human experience, this process continues with the comparison of each new interview or account until all have been compared with each other. A good example of this process is reported in a grounded theory study of how adults with brain injury cope with the social attitudes they face (see Evidence-Based Nursing, April 1999, p64).

Constant comparison analysis is well suited to grounded theory because this design is specifically used to study those human phenomena for which the researcher assumes that fundamental social processes explain something of human behaviour and experience, such as stages of grieving or processes of recovery. However, many other methodologies draw from this analytical strategy to create knowledge that is more generally descriptive or interpretive, such as coping with cancer, or living with illness. Naturalistic inquiry, thematic analysis, and interpretive description are methods that depend on constant comparative analysis processes to develop ways of understanding human phenomena within the context in which they are experienced.

\section{PHENOMENOLOGICAL APPROACHES}

Constant comparative analysis is not the only approach in qualitative research. Some qualitative methods are not oriented toward finding patterns and commonalities within human experience, but instead seek to discover some of the underlying structure or essence of that experience through the intensive study of individual cases. For example, rather than explain the stages and transitions within grieving that are common to people in various circumstances, a phenomenological study might attempt to uncover and describe the essential nature of grieving and represent it in such a manner that a person who had not grieved might begin to appreciate the phenomenon. The analytic methods that would be employed in these studies explicitly avoid cross comparisons and instead orient the researcher toward the depth and detail that can be appreciated only through an exhaustive, systematic, and reflective study of experiences as they are lived.

Although constant comparative methods might well permit the analyst to use some pre-existing or emergent theory against which to test all new pieces of data that are collected, these more phenomenological approaches typically challenge the researcher to set aside or "bracket" all such preconceptions so that they can work inductively with the data to generate entirely new descriptions and conceptualisations. There are numerous forms of phenomenological research; however, many of the most popular approaches used by nurses derive from the philosophical work of Husserl on modes of awareness (epistemology) and the hermeneutic tradition of Heidegger, which emphasises modes of being (ontology). ${ }^{5}$ These approaches differ from one another in the degree to which interpretation is acceptable, but both represent strategies for immersing oneself in data, engaging with data reflectively, and generating a rich description that will enlighten a reader as to the deeper essential structures underlying a particular human experience. Examples of the kinds of human experience that are amenable to this type of inquiry are the suffering experienced by individuals who have a drinking problem (see Evidence-Based Nursing, October 1998, p134) and the emotional experiences of parents of terminally ill adolescents (see Evidence-Based Nursing, October 1999, p132). Sometimes authors explain their approaches not by the phenomenological position they have adopted, but by naming the theorist whose specific techniques they are borrowing. Colaizzi and Giorgi are phenomenologists who have rendered the phenomenological attitude into a set of manageable steps and processes for working with such data and have therefore become popular reference sources among phenomenological nurse researchers.

\section{ETHNOGRAPHIC METHODS}

Ethnographic research methods derive from anthropology's tradition of interpreting the processes and products of cultural behaviour. Ethnographers documented such aspects of human experience as beliefs, kinship patterns and ways of living. In the healthcare field, nurses and others have used ethnographic methods to uncover and record variations in how different social and cultural groups understand and enact health and illness. An example of this kind of study is an investigation of how older adults adjust to living in a nursing home environment (see Evidence-Based Nursing, October 1999, p136). When a researcher claims to have used ethnographic methods, we can assume that he or she has come to know a culture or group through immersion and engagement in fieldwork or participant observation and has also undertaken to portray that culture through text. ${ }^{6}$ Ethnographic analysis uses an iterative process in which cultural ideas that arise during active involvement "in the field" are transformed, translated, or represented in a written document. It involves sifting and sorting through pieces of data to detect and interpret thematic categorisations, search for inconsistencies and contradictions, and generate conclusions about what is happening and why.

\section{NARRATIVE ANALYSIS AND DISCOURSE ANALYSIS}

Many qualitative nurse researchers have discovered the extent to which human experience is shaped, transformed, and understood through linguistic representation. The vague and subjective sensations that characterise cognitively unstructured life experiences take on meaning and order when we try to articulate them in communication. Putting experience into words, whether we do this verbally, in writing, or in thought, transforms the actual experience into a communicable representation of it. Thus, speech forms are not the experiences themselves, but a socially and culturally constructed device for creating shared understandings about them. Narrative analysis is a strategy that recognises the extent to which the stories we tell provide insights about our lived experiences. ${ }^{7}$ For example, it was used as a strategy to learn more about the experiences of women who discover that they have a breast lump (see Evidence-Based Nursing, July 1999, p93). Through analytic processes that help us detect the main narrative themes within the accounts people give about their lives, we discover how they understand and make sense of their lives.

By contrast, discourse analysis recognises speech not as a direct representation of human experience, but as an explicit linguistic tool constructed and shaped by numerous social or 
General distinctions between selected qualitative research approaches: an illustration using breast cancer research

\begin{tabular}{|c|c|c|c|}
\hline Method & $\begin{array}{l}\text { Research } \\
\text { question }\end{array}$ & $\begin{array}{l}\text { Analytic } \\
\text { strategy }\end{array}$ & Research product \\
\hline Grounded theory & $\begin{array}{l}\text { How do women } \\
\text { with breast } \\
\text { cancer cope } \\
\text { with changes to } \\
\text { body image? }\end{array}$ & $\begin{array}{l}\text { Constant } \\
\text { comparative } \\
\text { analysis }\end{array}$ & $\begin{array}{l}\text { Theory regarding } \\
\text { basic social } \\
\text { processes involved } \\
\text { in coping with breast } \\
\text { cancer and factors } \\
\text { that might account } \\
\text { for variations }\end{array}$ \\
\hline Phenomenology & $\begin{array}{l}\text { What is the } \\
\text { lived experience } \\
\text { of having breast } \\
\text { cancer? }\end{array}$ & $\begin{array}{l}\text { Phenomenological } \\
\text { reduction; } \\
\text { hermeneutic } \\
\text { analysis }\end{array}$ & $\begin{array}{l}\text { Description of the } \\
\text { essential structure } \\
\text { of breast cancer } \\
\text { experience }\end{array}$ \\
\hline Ethnography & $\begin{array}{l}\text { How is breast } \\
\text { cancer } \\
\text { understood and } \\
\text { managed in } \\
\text { different social } \\
\text { contexts? }\end{array}$ & $\begin{array}{l}\text { Representation, } \\
\text { inscription, } \\
\text { translation, and } \\
\text { textualisation of } \\
\text { culture into } \\
\text { writing }\end{array}$ & $\begin{array}{l}\text { Typology of } \\
\text { interpretations, } \\
\text { relations, and } \\
\text { variations within } \\
\text { breast cancer } \\
\text { experience }\end{array}$ \\
\hline Narrative analysis & $\begin{array}{l}\text { How do women } \\
\text { with breast } \\
\text { cancer come to } \\
\text { know their } \\
\text { experience? }\end{array}$ & $\begin{array}{l}\text { Generating, } \\
\text { interpreting, and } \\
\text { representing } \\
\text { women's stories } \\
\text { in narrative form }\end{array}$ & $\begin{array}{l}\text { Narrative accounts } \\
\text { of women's } \\
\text { explanations for } \\
\text { their breast cancer } \\
\text { experiences }\end{array}$ \\
\hline
\end{tabular}

ideological influences. Discourse analysis strategies draw heavily upon theories developed in such fields as sociolinguistics and cognitive psychology to try to understand what is represented by the various ways in which people communicate ideas. They capitalise on critical inquiry into the language that is used and the way that it is used to uncover the societal influences underlying our behaviours and thoughts. ${ }^{8}$ Thus, although discourse analysis and narrative analysis both rely heavily on speech as the most relevant data form, their reasons for analysing speech differ. The table illustrates the distinctions among the analytic strategies described above using breast cancer research as an example.

\section{Cognitive processes inherent in qualitative analysis}

The term "qualitative research" encompasses a wide range of philosophical positions, methodological strategies, and analytical procedures. Morse ${ }^{1}$ has summarised the cognitive processes involved in qualitative research in a way that can help us to better understand how the researcher's cognitive processes interact with qualitative data to bring about findings and generate new knowledge. Morse believes that all qualitative analysis, regardless of the specific approach, involves:

- comprehending the phenomenon under study

- synthesising a portrait of the phenomenon that accounts for relations and linkages within its aspects

- theorising about how and why these relations appear as they do, and

- recontextualising, or putting the new knowledge about phenomena and relations back into the context of how others have articulated the evolving knowledge.
Although the form that each of these steps will take may vary according to such factors as the research question, the researcher's orientation to the inquiry, or the setting and context of the study, this set of steps helps to depict a series of intellectual processes by which data in their raw form are considered, examined, and reformulated to become a research product.

\section{Quality measures in qualitative analysis}

It used to be a tradition among qualitative nurse researchers to claim that such issues as reliability and validity were irrelevant to the qualitative enterprise. Instead, they might say that the proof of the quality of the work rested entirely on the reader's acceptance or rejection of the claims that were made. If the findings "rang true" to the intended audience, then the qualitative study was considered successful. More recently, nurse researchers have taken a lead among their colleagues in other disciplines in trying to work out more formally how the quality of a piece of qualitative research might be judged. Many of these researchers have concluded that systematic, rigorous, and auditable analytical processes are among the most significant factors distinguishing good from poor quality research. ${ }^{9}$ Researchers are therefore encouraged to articulate their findings in such a manner that the logical processes by which they were developed are accessible to a critical reader, the relation between the actual data and the conclusions about data is explicit, and the claims made in relation to the data set are rendered credible and believable. Through this short description of analytical approaches, readers will be in a better position to critically evaluate individual qualitative studies, and decide whether and when to apply the findings of such studies to their nursing practice.

SALLY THORNE, RN, PhD School of Nursing, University of British Columbia Vancouver, British Columbia, Canada

1 Morse JM. "Emerging from the data": the cognitive processes of analysis in qualitative inquiry. In: JM Morse, editor. Critical issues in qualitative research methods. Thousand Oaks, CA: Sage, 1994:23-43.

2 Holloway I. Basic concepts for qualitative research. Oxford: Blackwell Science, 1997.

3 Schwandt TA. Qualitative inquiry: a dictionary of terms. Thousand Oaks, CA: Sage, 1997.

4 Glaser BG, Strauss AL. The discovery of grounded theory. Hawthorne, NY: Aldine, 1967.

5 Ray MA. The richness of phenomenology: philosophic, theoretic, and methodologic concerns. In:J M Morse, editor. Critical issues in qualitative research methods. Thousand Oaks, CA: Sage, 1994:117-33.

6 Boyle JS. Styles of ethnography. In:JM Morse, editor. Critical issues in qualitative research methods.. Thousand Oaks, CA: Sage, 1994:159-85.

7 Sandelowski M. We are the stories we tell: narrative knowing in nursing practice. J Holist Nurs 1994;12:23-33.

8 Boutain DM. Critical language and discourse study: their transformative relevance for critical nursing inquiry. ANS Adv Nurs Sci 1999;21:1-8.

9 Thorne S. The art (and science) of critiquing qualitative research. In: JM Morse, editor. Completing a qualitative project:details and dialogue. Thousand Oaks, CA: Sage, 1997:117-32. 